\title{
Editorial
}

\section{How many ways to die? How many different models of cell death?}

\author{
G Melino $^{*, 1,2}$, RA Knight ${ }^{1}$ and P Nicotera ${ }^{1}$ \\ ${ }^{1}$ Medical Research Council, Toxicology Unit, Hodgkin Building, Leicester \\ University, Lancaster Road, PO Box 138, Leicester LE1 9HN, UK \\ 2 Biochemistry Laboratory, IDI-IRCCS, c/o/Department Experimental Medicine, \\ University of Rome Tor Vergata, Rome 00133 , Italy \\ We wish to dedicate this manuscript to Peter M Steinert, whose sudden and \\ premature loss deprived us all of a great master and teacher. \\ * Corresponding author: G Melino, Medical Research Council, Toxicology Unit, \\ Hodgkin Building, Leicester University, Lancaster Road, PO Box 138, Leicester \\ LE1 9HN, UK. Tel: + 441162525551 ; Fax: + 441162525616 ; \\ E-mail: gm89@le.ac.uk
}

\begin{abstract}
A very late summer afternoon, becoming dark, after a very long days work in an obscure field in the English Midlands near a brewery. Three rats (all, of course, from the well known upper class Sprague-Dawley family) are drinking a well-earned beer, .... one is strongly inhaling a fag, another playing with his nuts, the last just trying to pile up papers for the next issue of the Midland rat's chronicle...
\end{abstract}

\section{Cell Death and Differentiation (2005) 12, 1457-1462. doi:10.1038/sj.cdd.4401781}

GM: So, Dick, according to the literature, apoptosis is a developmental remodelling programme and a defensive, organised self-destruction of the cell in reaction to severe damage. Unlike necrosis, where an uncontrolled release of internal cellular material results in the propagation of cell death and inflammation, apoptosis occurs inside an intact plasma membrane, thus preventing the release of intracellular contents. Recently, many reports have indicated the existence of distinct forms of cell death, such as 'caspaseindependent apoptosis', anoikis or excitotoxicity. Despite the fact that these share some common features, they are profoundly distinct processes (although they all end up effectively as death) occurring at different locations within the organism. Older evidence, subsequently forgotten, but more recently actively revitalised, indicated a further specific mechanism of programmed cell death - autophagy. But how many genetic programmes of cell death exist? I would also like to reflect on whether these programmes diverge from a common ancestral process or if they are totally unrelated. Is 'apoptosis' this ancestor in the Midland field?

In addition, the name of 'death' carries all sorts of ethical, religious and indeed evolutionary connotations, which inadvertently resonate with the scientific term, although attempts have been made, as elsewhere in this issue, to provide a clearer scientific definition (Figure 1). ${ }^{1}$ Now a crucial problem to discuss is the need to pose new questions in order to solve old problems.

RAK: In several reviews published in this issue of Cell Death Differentiation, several manuscripts describe autophagy in detail, ${ }^{2-10}$ while Lippens et al..$^{11}$ contrast two distinct forms of death, apoptosis and cornification in the skin, see also for review. ${ }^{12}$ Although these two processes may appear to have superficial similarities, their detailed mechanisms are largely different. ${ }^{12-14}$ For example, classical apoptosis, mainly affecting cells in the basal layer of the epidermis, and involving both extrinsic and intrinsic pathways, occurs following UVB irradiation. In contrast, cornification is spatially distinct, occurring in the suprabasal layers, and is a physiological process independent of external stimuli. While there is a limited overlap of the molecules involved in the two processes, the molecular executioners of apoptosis and cornification are, in general, different, although the molecular players in apoptosis induction are less well understood than those of cornification. ${ }^{12}$ However, the pathways leading to chromatin condensation and DNA fragmentation in apoptosis are well established; those resulting in nuclear loss in corneocytes are not. The mechanisms of anoikis ${ }^{15}$ are also discussed. Not least, the Nomenclature Committee on Cell Death (NCCD) has decided to reopen the whole nomenclature question in a discussion in this issue. ${ }^{16}$

The intense focus on apoptosis, and its disturbance in a wide range of human pathologies, has, perhaps, blinded us to the significance of other forms of cell death (and their particular pathologies), and this special issue of Cell Death Differentiation will, we hope, be useful in putting apoptosis into a broader context.

GM: How many different mechanisms of programmed cell death exist?

RAK: I think it is possible to discriminate up to 11 pathways of cell death occurring in mammals (Figure 2), 10 of which are genetically programmed. The different properties are shown based on the events occurring in particular cellular organelles. This classification is necessarily somewhat arbitrary, and largely based on the description of morphological events, but this in itself may suggest distinct molecular mechanisms.

Necrosis occurs predominantly in pathological conditions, such as myocardial and brain ischaemia. It is clearly not genetically programmed, but is the end point of very severe toxic damage. Necrosis can be secondary to apoptosis, for example in the presence of conflicting signals. The switch between necrosis and apoptosis can occur in the presence of nitric oxide-driven reactions, ${ }^{17,18}$ or conflicting regulation at the death-inducing signalling complex (DISC) in death receptor signalling. ${ }^{19,20}$ This is partially discussed in this same issue ${ }^{21}$ and reminds us of the original role of mitochondrial ATP levels in regulating the balance between apoptosis and necrosis in neuronal death. ${ }^{22,23}$

PN: I see the problem in an entirely different light. After all, apoptosis and programmed cell death are conceptually distinct entities. Apoptosis is a morphological phenotype of cell death, whereas programmed cell death refers specifically to a cell autonomous genetic developmental death programme. Apoptosis in mammalian cells can be executed in 
most cases via machinery that has strong homology with that described by Bob Horvitz and colleagues in Caenorhabditis elegans. However, in some circumstances part of this programme is dispensable and apoptotic-looking death can be executed independently of caspases. The morphology of 'caspase-independent' death is in fact not that different from cell death mediated by caspases. Neurons undergoing excitotoxic death (i.e., after exposure to high concentrations of glutamate) transiently swell, then shrink and their nucleus condenses; eventually they also display annexin- $V$, phosphatidylserine positivity. If an observer does not use multiple assays to link a particular morphological stage to an execution system (e.g. the caspases) the morphology alone can be misleading. Also accidental death caused, for example, by ischaemia in heart and brain can occur via the apoptotic machinery. So I think that this distinction is now redundant. My view is that cells doomed to die inevitably will have to process their intracellular organelles, the nucleus and cytoskeleton and will engage whichever execution system to that end. In development, the programme is genetically regulated and encoded. In adult cells the programme refers more to a defined series of biological processes that dismantle dying cells. In that sense, all forms of cell death are indeed programmed. Rather than classifying or subdividing cell death according to appearances, I am convinced that we should perhaps consider that cells can engage multiple death

\section{Different types of "Cell Death"}

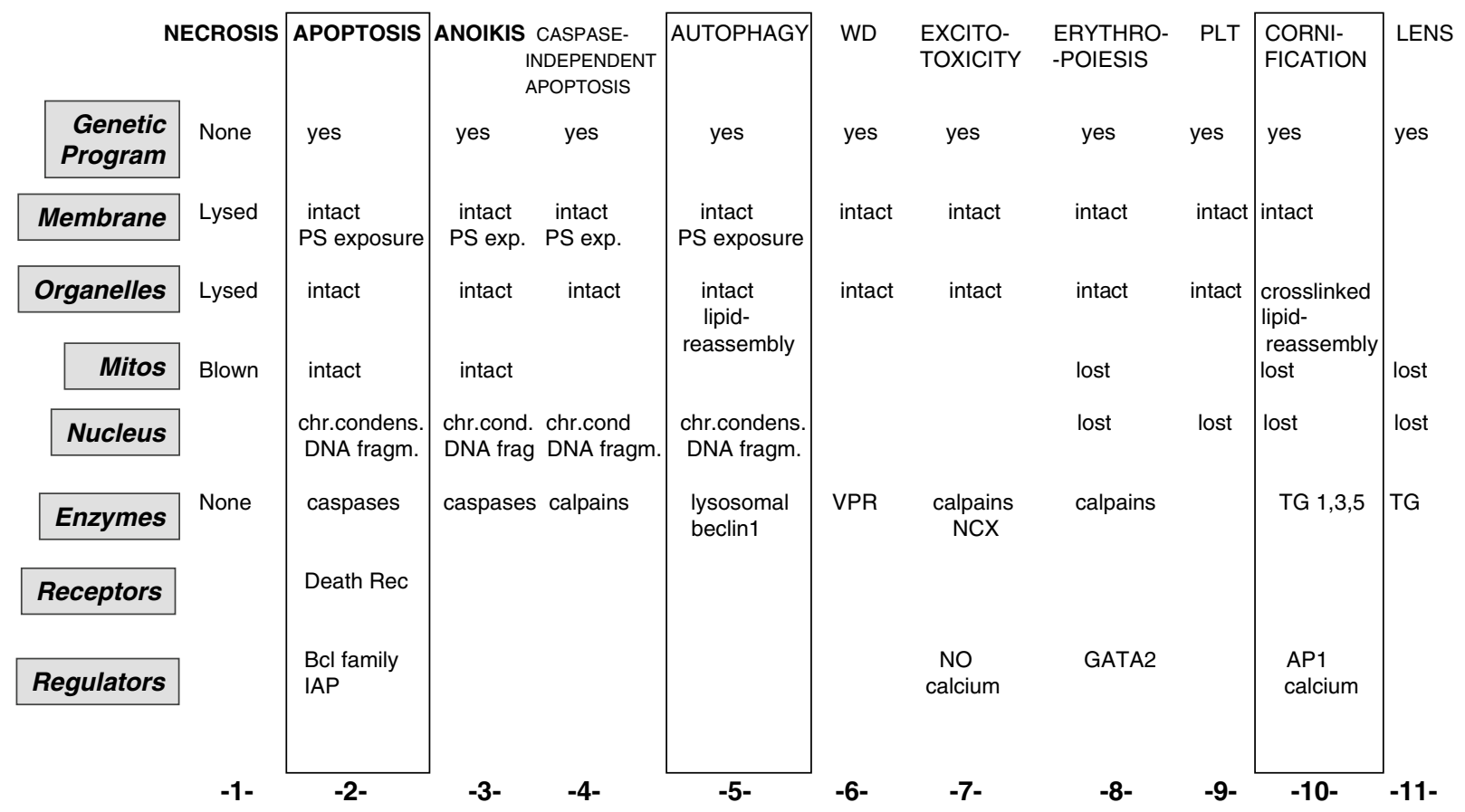

Figure 2 Different types of programmed cell death. Morphologically distinct types of cell death. At least 11 different types of cell death are known, 10 of which procede according to genetically programmed mechanisms. Some forms of death are not considered separately since they are identical to those indicated, that is, oncosis is a form of necrosis, and anoikis is apoptosis triggered by cell detachment. This does not exclude the possibility of additional mechanisms in higher or lower organisms such as in bacteria and Dictyostelium. The columns indicate, in a very simplified and schematic way, the different characteristics of the different forms of death, and in particular the findings in the plasma membrane, the nucleus, the mitochondria, and other cytosolic organelles. The details are only indicative, and are presented especially to highlight the differences between apoptosis, autophagy and cornification (boxes), which are discussed in detail in this special issue of Cell Death Differentiation. WD, Wallerian degeneration; PLT, platelets; TG, transglutaminases; NO, nitric oxide; NCX, sodium calcium exchange channel; IAP, inhibitor of apoptosis proteins 
routines, some being more efficient than others depending on the cell type (Figure 3 ). In fact the execution of cell death is different depending on the differentiation programme, and while the death machinery exists in virtually every cell, it might be used differently.

RAK: Indeed. But I don't think we want to describe all the different types of cell death in detail here, just highlight a few points. Apoptosis should not need a specific introduction, but in the unlikely event that any reader of CDD needs reminding of its basic mechanisms, there are several comprehensive reviews. ${ }^{24}$ Its role in development and in defence against insults, its involvement in human pathology, and the attempt to develop apoptosis-based therapy are also better described elsewhere. $^{24}$

PN: Dick, Could we for a moment forget the various 'types' of cell death and imagine that - when dying - cells simply may engage different execution routines (Figure 3)? The large majority of the key fundamental studies in apoptosis have used lymphoid cells, which display a predominant apoptotic phenotype and execution (try to push a thymocyte to undergo necrosis!). Authophagic cell death is instead studied in neurons, or other cells more prone to activate this programme. The error is to generalise the findings to all cell types. The machinery is everywhere but the execution may differ. For example, in brain ischaemia a subset of neurons die quickly, activating a programme that involves calpains, $\mathrm{Ca}^{2+}$ overload and downstream mitochondrial disruption without caspase activaton, ${ }^{25}$ but another subset can die by caspase activation with either an apoptotic or a necrotic morphology. If we stay away from a classification of cell death we may understand better some of the executing routines (a set of biochemically linked catabolic events that lead to cell processing). For example, it is not surprising that calpains and caspases share many substrates. It is also not surprising that cells are endowed with multiple proteolytic systems. A single or predominant execution pathway could be easily hijacked by viruses or inactivated with catastrophic consequences. I also cannot suppress a smile when I see articles that now focus intensively on caspase-independent cell death as if it represents a novelty. ${ }^{26}$ The field of caspase-independent cell death flourished before caspases were discovered. Thus, $\mathrm{Ca}^{2+}$-dependent cell death in brain and heart ischaemia has been known and studied for at least 40 years and understanding some of the fundamental signalling systems involved has led to very important therapeutic breakthroughs: just think of the $\mathrm{Ca}^{2+}$ channel blockers.

GM: As you know, I was a great friend and admirer of the late Peter Steinert and I would like to focus our discussion on two types (or subroutines if you wish, Pierluigi) of death occurring in the skin. ${ }^{12}$ Cornification is a specific mechanism of terminal differentiation and death of keratinocytes, occurring in some specialised epithelia, called cornified epithelia. It is also called keratinisation and cornified envelope (CE) formation. Curiously enough, this mechanism of programmed cell death is little regarded by scientists working on cell death, certainly as compared to apoptosis, despite the fact that it is well understood at the molecular level. ${ }^{12}$ I would like, therefore, to consider its relationship to apoptosis in a bit more detail.

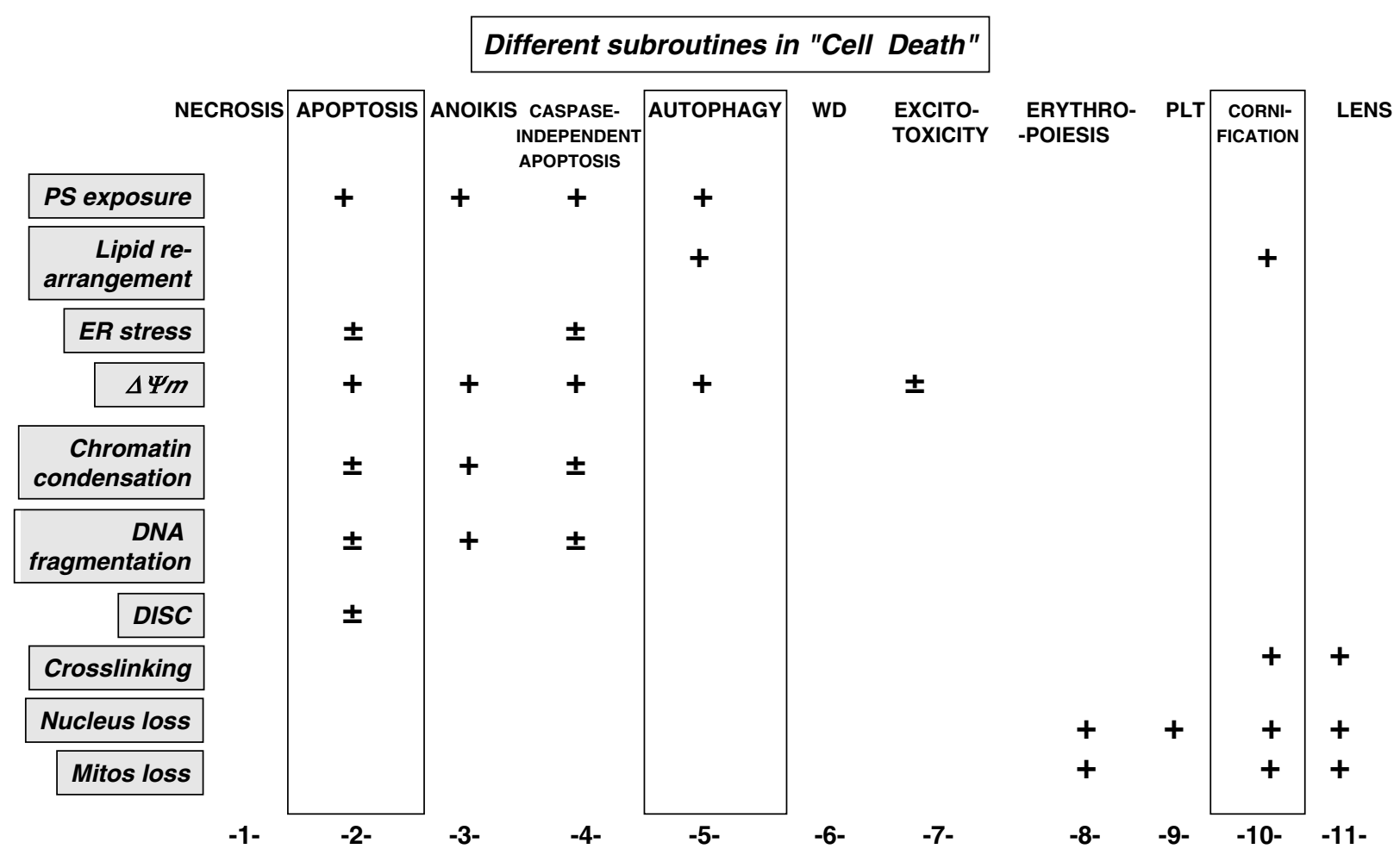

Figure 3 Different subroutines of programmed cell death. The same classification shown in Figure 2 can be reclassified as a mixture of different subroutines. This, however, requires additional experimental evidence to be fully comprehensive. ER, endoplasmic reticulum; PS, phosphatidylserine; DISC, death-initiating signalling complex 
The epidermis functions as a barrier against the environment by means of several layers of terminally differentiated, dead keratinocytes - the cornified envelope. ${ }^{12} \mathrm{CE}$ consists of keratins enclosed within an insoluble amalgam of proteins and lipids. Transglutaminases catalyse the formation of characteristic crosslinks between structural proteins to form the CE. Apoptosis also occurs in the skin. The molecular mechanisms and the physiological end points of apoptosis and cornification are profoundly different. In a very simplified way, defects of apoptosis seem to be preferentially related to the development of cancer, whereas CE abnormalities seem to be associated with barrier malfunctions and ichthyosis. ${ }^{12}$

In human skin, apoptosis occurs primarily in the basal layer of the epidermis in association with cell damage (e.g., in lichenoid disease, psoriasis and after ultraviolet irradiation). Both keratinocyte terminal differentiation and apoptosis are molecular programmes that result in cell death.

ANONYMOUS REFEREE 1: Because of certain similarities between the two processes, it has been suggested that terminal cell differentiation of epidermal keratinocytes is a specialised form of apoptosis. ${ }^{27,28}$ The similarities and differences are not evident.

GM: Thank you for your constructive suggestion.

RAK: Perhaps then, Gerry, you could summarise the similarities and differences between cornification and apoptosis.

GM: OK, I'll do so in Table 1. First, the similarities. For both apoptosis and keratinocyte differentiation, the interaction with the extracellular matrix through integrins is important. Keratinocytes will start to differentiate when contact with the basal membrane is lost, and indeed keratinocytes can be induced to differentiate in vitro by forced suspension growth. In contrast, other cell types will die by apoptosis when they become detached from the substrate in a process known as anoikis (e.g., see Gilmore ${ }^{15}$ and Frisch and Francis ${ }^{29}$ ). The transcription factor c-myc has also been shown to be involved in both processes. In certain cells the constitutive activation of c-myc results in apoptosis, ${ }^{30}$ whereas overexpression of c-myc in keratinocytes induces terminal differentiation. ${ }^{31}$ The bcl-2 family members, in particular Bcl-2 itself, are downregulated during terminal differentiation, whereas expression of the proapoptotic proteins Bax and Bak is still found in the suprabasal layers of the epidermis. ${ }^{32,33}$

PN: And, surely, to plug one of my favourite areas, increases in $\mathrm{Ca}^{2+}$ concentration induce both keratinocyte differentiation and cell death, although, in apoptosis, $\mathrm{Ca}^{2+}$ may also have other roles than a direct trigger. ${ }^{34,35}$

GM: I agree. As to the differences. Despite the similarities I've described above, growing evidence suggests that keratinocyte differentiation and apoptosis occur through different molecular pathways. ${ }^{36}$ Apoptosis is a rapid process, all the components for signal transduction are present in the cells and no de novo protein synthesis is required to execute the apoptotic pathway. In contrast, keratinocyte differentiation is a slow, coordinated process in space and time, which requires the ordered production of typical differentiationassociated proteins. The plasma membrane during apoptotic body formation remains intact (membrane blebbing), whereas during keratinocyte differentiation the plasma membrane disappears after fusion with the lamellar bodies. ${ }^{12}$ The corneocytes, the final product of keratinocyte differentiation, fulfil an important physiological role before they desquamate into the environment, ${ }^{12}$ whereas the apoptotic cell has no obvious function and is eliminated by phagocytosis.

Both apoptosis and keratinocyte differentiation involve the destruction of the nucleus, although the mechanisms are distinct. Nuclear degradation during apoptosis is marked by chromatin condensation and DNA laddering, as detected by

Table 1 Common and distinctive features of cornification and apoptosis

\section{Common features}

Intrinsic control mechanism

Elimination of cells without either tissue destruction, inflammation or scar formation

Disintegration of DNA and nuclei

Modulation by glucocorticoids and retinoids

\begin{tabular}{|c|c|c|}
\hline Distinctive features & Cornification & Apoptosis \\
\hline $\begin{array}{l}\text { Elimination of dead cells by: } \\
\text { Cell organelles are: } \\
\text { Nucleus: } \\
\text { Chromatin fragmentation: } \\
\text { Plasma membrane: } \\
\text { Duration of the process: } \\
\text { Activation of the process by } \mathrm{Ca}^{2+} \text { ions: } \\
\text { Enzymes involved: } \\
\text { Enzyme substrates (examples): }\end{array}$ & $\begin{array}{l}\text { Desquamation } \\
\text { Lysed } \\
\text { Enlargement, } \\
\text { No } \\
\text { No DNA fragmentation } \\
\text { TUNEL negativity } \\
\text { Fused with lamellae } \\
\text { Slow (20 days) } \\
\text { Obligatory } \\
\text { Transglutaminases } \\
\text { Keratins } \\
\text { Loricrin } \\
\text { SPRs } \\
\text { Filaggrin } \\
\text { Trichohyalin } \\
\text { Involucrin } \\
\text { Cystatin } \alpha \\
\text { Elafin } \\
\text { Desmosomal proteins }\end{array}$ & $\begin{array}{l}\text { Phagocytosis } \\
\text { Encapsulated } \\
\text { Condensation } \\
\text { Yes } \\
\text { DNA fragmentation } \\
\text { TUNEL positivity } \\
\text { Intact } \\
\text { Rapid (20 min) } \\
\text { Facultative } \\
\text { Caspases, calpains } \\
\text { ICAD } \\
\text { Involucrin } \\
\text { Rb } \\
\text { Vinculin } \\
\text { IL-2 } \\
\text { PARP } \\
\text { Serca } \\
\text { p75 } \\
\text { Bid }\end{array}$ \\
\hline
\end{tabular}


TUNEL (terminal dUTP-biotin nick end labelling) staining. Although TUNEL staining has been sporadically observed in differentiating keratinocytes, ${ }^{37}$ the nuclei are enlarged rather than condensed and no chromatin condensation nor DNA laddering is detected. ${ }^{36}$

Caspases have an important role in apoptosis ${ }^{38}$ by cleaving a large number of substrates. ${ }^{39}$ Different caspases are expressed in the lower layers of the epidermis (caspases $1-4,6-9,11$ ), as compared to other layers (caspase 14). ${ }^{40}$ Participation of caspase-3 in corneocyte formation was suggested by one research group, ${ }^{41}$ although other investigators showed that caspase-3, as well as other caspases, remain unprocessed in differentiating keratinocytes and no abnormal skin formation has been reported in caspase-3-/14,42 or other caspase-deficient mice. On the other hand, caspase-14, which is not involved in apoptosis, is processed in the later stages of epidermal differentiation. ${ }^{14,40}$ Elucidation of its exact role requires the generation of knockout mice.

RAK and PN (joint lament!): So our verdict would go something like this; in human skin cell death occurs via two mechanisms: terminal keratinocyte differentiation, with a defined physiological function of the dead cells (corneocytes) constituting the skin barrier; and apoptosis, which occurs mainly in the basal layer as a defence reaction to eliminate heavily damaged cells and prevent propagation of nuclear damage to daughter progeny.

GM: Yes. The reasons are multifold. First, the major players in apoptosis do not seem to affect cornification; see for example, the studies on knockout animals. Similarly, the skin-related protein knockout mice do not have compromised apoptotic machinery. Second, defective cornification proteins cause specific skin diseases without any corresponding defects in apoptosis. Third, cornification appears in evolution only as a biological and molecular event related to the emergence of skin, and is therefore a much more recent process in evolutionary terms, perhaps as an essential adaptation to terrestrial life. Animals that lack skin (and cornification), such as C. elegans and Drosophilla melanogaster, still have a well-developed apoptotic machinery, as elegantly described by Jean-Claude Ameisen. ${ }^{43-45}$ Specific subroutines of cornification, namely the elimination of nucleus and mitochondria, also appear in different peculiar nonapoptotic forms of cell death, possibly suggesting a common origin (at least for some subroutines). Cornification and apoptosis are therefore an example of divergent evolution.

RAK: Gerry, you also mentioned that you wanted to use this discussion to propose new hypotheses. What do you propose?

GM: The hypothesis. I suggest that the first event of cornification is the suppression of apoptosis. While apoptosis eliminates unnecessary or damaged cells, cornification is a molecular preparation to allow cells to perform duties 'after death' (if we could use a philosophical and ethical term in a scientific context). As outlined in the present discussion, dead keratinocytes must be mechanically resistant, elastic and waterproof, and possess these properties to differing degrees in different regions of the body. To this end they need very specific molecular events and sophisticated mechanisms, and not a simple 'apoptotic death'. Indeed, apoptosis (and its molecules such as p53, bcl2, etc.) is seen only in the basal layer (e.g. after UV exposure). Many of the enzymes involved in cornification, and their substrates are extremely toxic, and their expression requires the suppression of the apoptotic machinery. The suppression of apoptosis by inhibitors and elimination of crucial molecules allows the free expression of these dangerous cornification-specific molecules that requires extremely tight regulation to avoid skin defects.

The final chorus: In this discussion we stress that there are many ways through which cells process themselves in the final stages of their life. ${ }^{1,12,24,43-45}$ Definitions of different forms or routines include: necrosis, apoptosis, cornification, Wallerian degeneration, caspase-independent apoptosis, platelet formation, erythropoiesis and autophagocytosis. Definitions have always attracted debates, as originally discussed in this journal, ${ }^{46,47}$ and in the present issue. ${ }^{16}$ We have concentrated more on the mechanisms of death occurring in the skin, on cornification, a sophisticated mechanism of cell death that is also essential for life. The molecular events involved are described elsewhere, ${ }^{24,48}$ and in particular, the role of transglutaminases and their substrates, with the related diseases caused by abnormalities in these proteins is detailed in more appropriate reviews. ${ }^{24,48}$ Here we have contrasted cornification with apoptosis, arguing for completely distinct molecular mechanisms. Many interesting, unresolved questions remain; for example, it is as yet unknown how the keratinocyte dies, or more specifically, what the precise suicide signal is, and how nucleus and mitochondria are eliminated.

[dancing, wind blowing]

From Figures 2 and 3 it is evident that there are distinct forms of programmed cell death. While much research effort over the last decade has been devoted to understanding classical apoptosis, other cell death mechanisms have been less favoured. It is now time for these other pathways to come into the limelight, and this will undoubtedly raise new problems and questions. Once the molecular pathways or routines of the whole spectrum of cell death processes are better understood, we shall be better placed to therapeutically manipulate their dysfunctions that result in so many human diseases.

Stop, stop, somebody is coming ... it must be Godot ....... Background voice (... the landlord): We dedicate this Editorial to Peter M Steinert, master and mentor to several of us, who died prematurely. We thank Doug Green and Gerry Cohen for helpful discussions and criticism which, of course, remains ongoing.

1. Melino G (2001) Nature 412: 23

2. Codogno $P$ and Meijer AJ (2005) Cell Death Differ. 12: 1509-1518

3. Deretic V (2005) Cell Death Differ. 12, this issue

4. Eskelinen EL (2005) Cell Death Differ. 12: 1468-1472

5. Golstein P and Kroemer G (2005) Cell Death Differ. 12: 1490-1496

6. Kundu M and Thompson CB (2005) Cell Death Differ. 12: 1484-1489

7. Mizushima N (2005) Cell Death Differ. 12: 1535-1541

8. Schmid D and Münz C (2005) Cell Death Differ. 12: 1519-1527

9. Tsujimoto $Y$ and Shimizu S (2005) Cell Death Differ. 12: 1528-1534

10. Yorimitsu T and Klionsky DJ (2005) Cell Death Differ. 12: 1542-1552

11. Lippens $S$ et al. (2005) Cell Death Differ. 12: 1497-1508

12. Candi E, Schmidt R and Melino G (2005) Nat. Rev. Mol. Cell Biol. 6: 328-340

13. Allombert-Blaise $C$ et al. (2003) Cell Death Differ. 10: 850-852

14. Lippens $S$ et al. (2000) Cell Death Differ. 7: 1218-1224 
33. Krajewski S et al. (1994) Am. J. Pathol. 145: 1323-1336

15. Gilmore A (2005) Cell Death Differ. 12: 1473-1477

16. Kroemer $\mathrm{G}$ et al. (2005) Cell Death Differ. 12: 1463-1467

17. Melino $G$ et al. (1997) Nature 388: 432-433

18. Nicotera $P$ and Melino G (2004) Oncogene 23: 2757-2765

19. Vercammen $D$ et al. (1998) J. Exp. Med. 188: 919-930

20. Matsumura $\mathrm{H}$ et al. (2000) J. Cell Biol. 151: 1247-1256

21. Zamzami N, Larochette N and Kroemer G (2005) Cell Death Differ. 12: $1478-1480$

22. Bonfoco $\mathrm{E}$ et al. (1995) Proc. Natl. Acad. Sci. USA 92: 7162-7166

23. Leist $\mathrm{M}$ et al. (1997) J. Exp. Med. 185: 1481-1486

24. Insight on Apoptosis (2000) Nature 407: 769-816

25. Bano D et al. (2005) Cell 120: 275-285

26. Kroemer $G$ and Martin SJ (2005) Nat. Med. 11: 725-730

27. Fesus $L$ and Thomazy V (1988) Adv. Exp. Med. Biol. 231: 119-134

28. Haake AR and Polakowska RR (1993) J. Invest. Dermatol. 101: 107-112

29. Frisch SM and Francis H (1994) J. Cell. Biol. 124: 619-626

30. Packham G and Cleveland JL (1995) Biochim. Biophys. Acta 1242: 11-28

31. Gandarillas A and Watt FM (1997) Genes Dev. 11: 2869-2882

32. Krajewska M et al. (1996) Am. J. Pathol. 149: 1449-1457
34. McConkey DJ, Orrenius S and Jondal M (1990) J. Immunol. 145: 1227-1230

35. Orrenius S, Zhivotovsky B and Nicotera P (2003) Nat. Rev. Mol. Cell Biol. 4: $552-565$

36. Gandarillas A et al. (1999) Exp. Dermatol. 8: 71-79

37. Polakowska RR et al. (1994) Dev. Dyn. 199: 176-188

38. Nicholson DW (1999) Cell Death Differ. 6: 1028-1042

39. Fischer U, Janicke RU and Sculze-Ostoff K (2003) Cell Death Differ. 10: $76-100$

40. Lippens $S$ et al. (2003) Cell Death Differ. 10: 257-259

41. Weil M, Raff MC and Braga VM (1999) Curr. Biol. 9: 361-364

42. Kuida $\mathrm{K}$ et al. (1996) Nature 384: 368-372

43. Ameisen JC (2004) Cell Death Differ. 11: 4-10

44. Ameisen JC (2002) Cell Death Differ. 9: 367-393

45. Ameisen JC (1999) La sculpture du vivant. Le suicide cellulaire ou la mort crèatrice. 4th ed. 2003, Paris: Editions du Seuil

46. Samali A et al. (1999) Cell Death Differ. 6: 495-496

47. Vaux DL (1999) Cell Death Differ. 6: 493-494

48. Okada H and Mak TW (2004) Nat. Rev. Cancer 4: 519-603 